\begin{tabular}{|c|c|c|}
\hline \multirow{2}{*}{ BENTHAM OPEN } & $\begin{array}{c}\text { The Open Cybernetics \& Systemics } \\
\text { Journal }\end{array}$ & $\begin{array}{l}\text { The Open } \\
\text { Chbernceics \& Systemics } \\
\text { loumal }\end{array}$ \\
\hline & $\begin{array}{l}\text { Content list available at: www.benthamopen.com/TOCSJ/ } \\
\text { DOI: } 10.2174 / 1874110 \mathrm{X} 01610010147\end{array}$ & 10 \\
\hline
\end{tabular}

RESEARCH ARTICLE

\title{
Seed Identification of Gramineous Grass Based on Difference of Local Fractal Dimensions
}

\author{
Xin Pan $^{*}, 1$, Tong Chen ${ }^{1}$, Yubao $\mathrm{Ma}^{2}$, Weihong Yan ${ }^{2}$, Xiaojing Gao ${ }^{1}$, Xia Liu ${ }^{1}$ and Guixiang Liu ${ }^{*} 2$ \\ ${ }^{1}$ College of Computer and Information Engineering, Inner Mongolia Agricultural University, Huhhot, Inner Mongolia \\ 010018, China \\ ${ }^{2}$ Grassland Research Institute of Chinese Academy of Agricultural Sciences, Huhhot, Inner Mongolia 010010, China
}

Received: November 24, 2015

Revised: June 27, 2016

Accepted: July 25, 2016

\begin{abstract}
This work proposes an approach based on the difference of local fractal dimension (DLFD) for seed identification of gramineous grass, rather than shape and color of the seeds. Being an important forge category of grassland, gramineous grass has been rarely investigated for the automated identification task by the researchers. Three main steps are involved in the extraction of DLFD. At first, the ROI image is equally divided into local blocks, and the fractal dimension of the partitions are calculated. Based on the average fractal dimension of all the blocks, the DLFD can then be obtained by subtracting the individual fractal dimension and the average, magnifying the contrast of the self-similarity of the images. Euclidean Distance and the nearest neighbor classifier are finally used for similarity measurement and classification. The novelty of the approach lies in applying fractal geometry in forage seed identification, a quite new area for pattern recognition. The experimental results demonstrate the effectiveness of the proposed method by some comparative analysis.
\end{abstract}

Keywords: Fractal dimension, Gramineous grass, Seed identification, Texture analysis.

\section{INTRODUCTION}

Grassland is the refreshable natural resource for human beings, and the dynamic monitor of grassland is vital for the sustainable strategy of the environment. Among all the elements of the grassland, forage plays the most important role in providing food for cattle and keeping balance of our ecosystem. Forage identification is the key approach for us to know and protect the grassland. However, the classical forage identification method is implemented manually by the experts with a low accuracy and efficiency. Sometimes, even the experts can not identify them until the grass bloom. To solve the above issues, it is necessary to develop an automatic system based on computer vision for efficient and accurate identification of forage.

Computer vision is a multi-disciplinary technology that integrates computer and image acquisition system to imitate the human vision in a procedure of digital image conversion, image transmission, image processing and image understanding. Weed detection and classification [1,2] aiming at the reduction of herbicide use and environmental pollution [3] have been successfully implemented by computer vision, which accumulate precious experience for forage identification. Owing to the seeds are more stable and rarely affected by their surroundings, the unique structures and appearances have become the important features to be investigated. Pablo M. Granitto et al. [4, 5] assessed the discriminating power of 57 weed seed species. The 12 feature vectors were composed of six morphological, four color and two textural seed characteristics, and Bayes Classifier were testified surprisingly good for the classification performance. The recognition rates reached $99.3 \%$ and $98.2 \%$ tested by a large scale seed base containing 10,310 images of 236 different weed species with color images and black and white seed images, respectively.

\footnotetext{
* Address correspondence to these authors at the College of Computer and Engineering, Inner Mongolia Agricultural University, 306Road ZhaoWuda, Saihan District, Huuhot, Inner Mongolia, China; Tel: 86-15847129078; E-mails: pxffyfx@126.com, liugx804@163.com
} 
Shi et al. [6] proposed a seed identification system for leguminous weeds. In their work, the feature vector composed of 16 components includes the shape geometry and the inner structures of the seeds and umbilicus. The concrete features include the major axis, surface area, perimeter, position and angle between the center points, and $7 \mathrm{Hu}$ invariant moments. The BP Network and SVM classifier are used for classification, yielding a recognition accuracy of $89.29 \%$, using a seed database comprised by 5,181 microscope images of 808 species.

The above researches mainly extract the features of appearance and shapes for relatively obvious difference among large numbers of weeds species of our ecosystem. However, when we focus on seed identificaiton and quality assessment of forage, it is interesting to note that similiar seeds, especially in some close species of the same family (genus), are hard to be identified correctly, even by the experts. For example, gramineous grass, an important category in grassland forage and urban landscaping, has some similar varieties, such as Agropyron cristatum var. pectiniforme (Roem.et Schult) H Yang (Fig. 1), Agropyron cristatum (Linn.) Gaertn (Fig. 2), Agropyron desertorum (Fisch.) Schult. Fig. (3) and Agropyron mongolicum Keng (Fig. 4), etc., categorized to Agropyron Gaertn Genus. It can be observed that the previous experience can not be directly applied to seed identification of forage, for the varieties of same family are too similar to distinguish from the appearance. Texture analysis can be used for reference in similar seed identification. Pourreza et al. [7] attempted to extract textual features for similar varieties in wheat seed identification. They investigated 9 Iranian wheat seed varieties conducted on bulk sample images and extracted 131 textural features for identification including GLCM (gray level co-occurrence matrix), GLRM (gray level run-length matrix), LBP(local binary patterns), etc. LDA (linear discriminate analysis) classifier was employed for classification using top selected features, yielding an average classification accuracy of $98.15 \%$ with top 50 selected features.

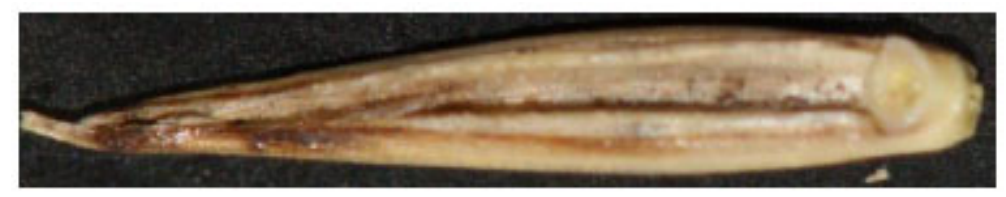

Fig. (1). Agropyron cristatum var.pectiniforme (Roem. \& Schult) H Yang.

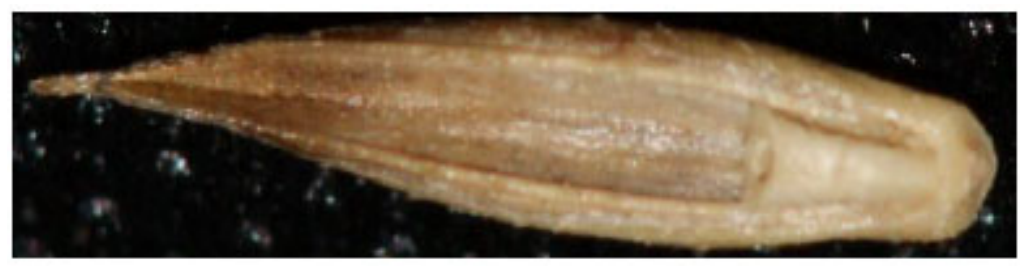

Fig. (2). Agropyron cristatum (Linn.) Gaertn.

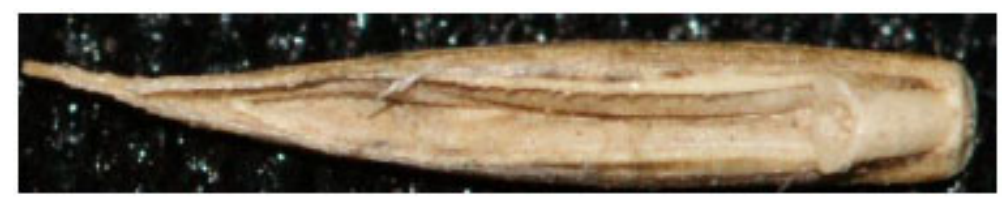

Fig. (3). Agropyron desertorum (Fisch.) Schult.

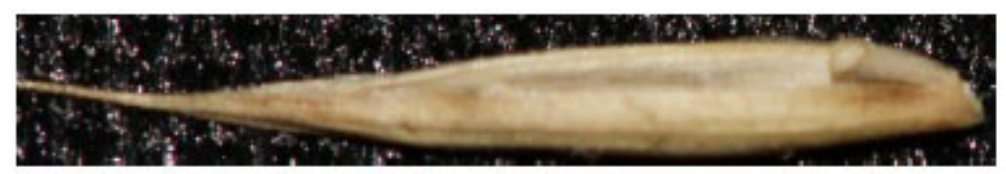

Fig. (4). Agropyron mongolicum Keng.

In texture analysis, fractal geometry is a powerful modeling tool, achieving interesting results in the description and discrimination of textures [8]. The surface texture of seed have local self-similarity and fractal dimension can measure the roughness and self-similarity of the shape and texture [9]. Therefore, in this work, we make our efforts for more discriminant features by calculating the difference of fractal dimensions of the local blocks for seed identification of similar forage species. Being one of the most important kinds of forage in grassland, gramineous grass plays a vital role 
in providing the food resource for the livestock and keeping balance of the bio-system of grassland for human being. Moreover, gramineous grass is also the important kind of lawn grass for urban landscape. So in this work, we focus on the seed identification method for gramineous grass.

The identification algorithm can be divided into 3 steps. First, the ROI images cropped from the original seed image are divided into blocks of same size for local partitions, and the fractal dimensions of all the blocks are calculated. Then, based on the average fractal dimension of all the blocks, the difference of local fractal dimension (DLFD) can be gained by subtracting the individual fractal dimension and the average, expanding the contrast of the self-similarity of the seeds. The above DLFDs of all blocks form the feature vectors for seed image representation. Finally, we calculate the Euclidean Distances as the input of nearest neighbor classifier for classification.

The rest of this paper is organized according to the sequence of identification as follows. Section 2 introduces the fractal dimension. Section 3 mainly focuses on the feature extraction, difference of local fractal dimension algorithms, and feature matching is also involved. Experimental results are listed in Section 4. Section 5 highlights the conclusion.

\section{FRACTAL AND DIMENSION}

Fractal geometry of nature was first proposed by Mandelbrot to describe the objects exhibiting the self-similarity at all scales [10]. The concept of fractal dimension (FD) is widely used for texture analysis and classification and results in good performance $[11,12]$. FD is a simple indicator of measuring the roughness and self-similarity of shape and texture. Mandelbrot defined fractal dimension D of A by the following equation [10].

$$
D=\frac{\log \left(N_{r}\right)}{\log (1 / r)} .
$$

For a bounded set $A$ in Euclidean n-space, if $A$ is the union of $N r$ non-overlapping distinct copies of itself each of which is similar to $A$ scaled down by a ratio $r$, the set is self-similar. However, it is very difficult to compute $D$ directly. Sarkar and Chaudhuri [9] developed a simple, accurate and efficient algorithm named differential box counting (DBC) for the estimation of FD. In their method, an image of size $\mathrm{M} \times \mathrm{M}$ is scaled down to a size s $\times \mathrm{s}$ where $\mathrm{s}$ is an integer ${ }^{M} / 2 \geq$ $s>1$, then ratio $\mathrm{r}=\mathrm{s} / \mathrm{M}$. Considering the image as a 3-D space with coordinates $(x, y)$ and $(z)$ denoting 2-D position and gray level respectively, the image is partitioned into grids by the $s \times{ }_{S} \times s$ boxes. If the minimum and the maximum gray level of the $(i, j)$ th grid fall in the box number $k$ and $l$ respectively, the contribution of $N r$ in the $(i, j)$ th grid is:

$$
n_{r}(i, j)=l-k+1 \text {. }
$$

And the total contributions $\mathrm{Nr}$ of all the grids is:

$$
N_{r}=\sum_{i, j} n_{r}(i, j)
$$

then the FD at a scale $\mathrm{r}$ can be computed by Eq. (1).

\section{DIFFERENCE OF LOCAL FRACTAL DIMENSION (DLFD)}

Although fractal dimension excels at describing the richness texture of a complex image, and scale-dependent [8], it is still hard to precisely represent a whole image with the only fractal dimension. Local matching partitioning the region of interest (ROI) into smaller sub-images can bind the effects of image variations and better preserve local information [13]. Therefore, in this work, ROI seed image is divided into local partitions whose fractal dimensions are used for local information presentation.Considering that the fractal dimensions vary in a certain range, the differences of all blocks and their average form feature vectors to magnify the local variations.

Suppose an image $I(x, y)$ of size $M \times N$, the partition is to divide palmprint image into non-overlapping uniform subblocks of size $2^{s} \times 2^{s}$. Due to the contrast of minimum and the maximum gray level during caculation procecedure of fractal dimension using BCD, the minmum block should be at least $2^{2} \times 2^{2}$, that is should be the integers between 2 and $\log _{2} \min (M, N)$. Then the local fractal dimension of each block $\mathrm{A}_{\mathrm{pq}}$ can be calcuated as following

$$
\operatorname{LFD}\left(A_{p q}\right)=\sum_{i, j \in A_{p q}} n_{r}(i, j)
$$


where $p$ and $q$ are integers within the scope of $1 \leq p=M / 2^{s}, 1 \geq q=N / 2^{s}$. Considering that the values of the fractal dimension for all partitions are relatively close, the difference of the local fractal dimensions and their average are computed to magnify the contrast between local blocks. Meanwhile, the difference can alleviate the effect of illumination variation by subtracting the average fractal dimension. The average fractal dimension can be calculated as follows:

$$
\mu(F D)=\sum_{p=1}^{M / 2^{s}} \sum_{q=1}^{N / 2^{s}} \operatorname{LFD}\left(A_{p q}\right)
$$

Thus the difference of the local fractal dimension for each partition is:

$$
D L F D\left(A_{i j}\right)=F D\left(A_{i j}\right)-\mu(F D)
$$

Then, we can form a feature vector composed of DLFD of all the subblocks for image representation:

$$
F_{D L F D}=\left(D L F D\left(A_{11}\right), D L F D\left(A_{12}\right), \ldots, D L F D\left(A_{M / 2^{s} N / 2^{s}}\right)\right)
$$

Having normalized the feature vectors, the Euclidean distance and nearest neighbor classifier are used to measure and classify similarity for simplicity, respectively. The concrete equation of Euclidean distance is:

$$
d\left(F_{D L F D}(\text { test }), F_{D L F D}(\text { train })\right)=\sqrt{\sum_{p=1}^{M / 2^{s}} \sum_{q=1}^{N / 2^{s}}\left(F_{D L F D}(\text { test })_{p q}-F_{D L F D}(\text { train })_{p q}\right)^{2}}
$$

The nearest neighbor classifier is used for identification.

\section{RESULTS AND DISCUSSIONS}

\subsection{Seed Image Database}

To test the effectiveness of the proposed algorithm for seed identification, we set up an image database of the gramineous-grass seeds, which were provided by the Grassland Research Institute of the Chinese Academy of Agricultural Sciences. The database contained 950 gramineous seed images comprised of 19 species from 5 genera (Elymus, Agropyron, Bromus, Roegneria and Stipa). In addition to the first batch seed [14], 5 species of gramineous seeds were supplemented in the seed image database, including Roegneria stricta Keng, Elymus breviaristatus (Keng) Keng f., Leymus secalinus (Georgi) Tzvel., Roegneria ciliaris(Trin.)Nevski, and Roegneria turczaninovii (Drob.) Nevski. 10 seeds were collected for each species, and each of them was captured five times to reduce the effect caused by the variations of focuses, orientations and positions during image acquisition. Therefore, there were 19 classes in the database, and each class harbored 50 image samples taken from 10 seeds.

In the procedure of image acquisition, a gramineous grass seed was put on the surface center of a black mouse mat for less diffuse reflectance, captured by a commercial CCD camera (DSLR-A350, Sony) under natural daylight illumination inside a room without manual control. The other options were set automatically under the standard program of the camera, including auto flash and focus.

Three main steps were involved in image preprocessing for the Region of Interest (ROI). Initially, the original RGB color images were converted into gray images for the color of the gramineous seeds were mostly medium brownish. The images were then converted into its binary form with a proper threshold of gray levels. Subsequently, an open mathematical morphology operation [15], was applied to the binary image to eliminate the noises of the background. At last, the image was rotated to keep the principal axis horizontal, and the minimum enclosing rectangle of the seed was cropped as the ROI of the original image. Figs. (5 and 6) showed the original image and the ROI, respectively. The background regions were removed for less interference on the texture extraction Fig. (7). 


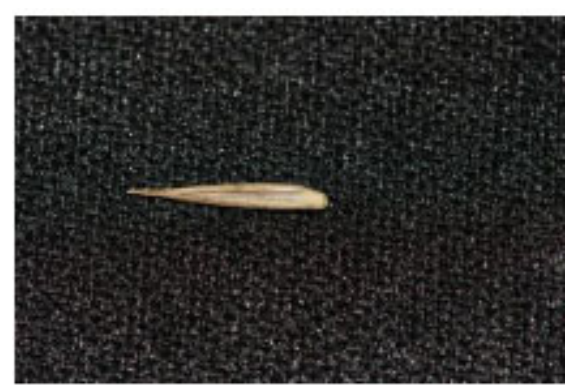

Fig. (5). Original seed image (Agropyron mongolicum Keng).

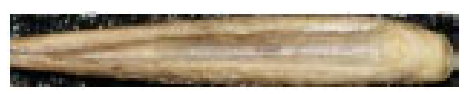

Fig. (6). ROI after segmentation.

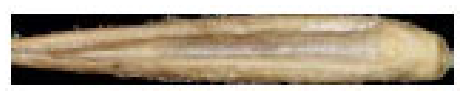

Fig. (7). ROI without background.

\subsection{Seed Identification}

To test the effectiveness of the algorithm, we carried out two groups of experiments, one was to determine the optimum parameters for division strategy, and the other was to compare the identification performance of different algorithms. All of the experiments were executed on an Intel Core i5-2467M CPU @ 1.60 GHz and 6 GB RAM; the codes were written in MATLAB 7.0.

\subsubsection{Local Division Strategy}

As we know, the ROIs cropped from original images are of different sizes because of the diversity in geometry size and shape of forage seeds, and auto-focusing without fixed camera equipment in image acquisition. For further local feature extraction, the ROI images should be normalized into a uniform size. Seeing that the outlines of gramineous seeds are mostly elliptical, the ratios of height to width were set from 1:1 to 1:10, with normalized resolutions from $64 \times 64$ to $64 \times 640$. In addition, the size of partition area is another important factor in local division strategy, which we set $64 \times 64,32 \times 32,16 \times 16,8 \times 8$ and $4 \times 4$, respectively. By comparing the identification ratios of LFD and DLFD, the optimum combination of normalized size and division area could be found.

Table 1 compares the identification accuracies and feature dimensions of LFD and DLFD at different normalized sizes and local partition areas. When the division area is relatively large, the local fractal dimensions of few local blocks can not well describe the texture of the image completely, yielding poor identification performance. Especially for a $64 \times 64$ normalized image with the partition area $64 \times 64$, the only feature reveals an identification accuracy of $26.11 \%$. That proves the global fractal dimension can not represent the whole image effectively. The DLFD is of no meaning because of no difference can be calculated between the only local fractal dimension and the average. The identification accuracies rise with the increase of normalized size and feature dimension, in which the identification performance of DLFD is not as good as that of LFD until the normalized size reaches $64 \times 384$. The identification accuracies of LFD and DLFD arrive at $67.58 \%$ and $71.58 \%$, respectively, with 6 feature components divided by $64 \times 64$ local partitions. The reason mainly lies in that the average of few partitions makes no sense as a reference of texture roughness.

For the local partition area $32 \times 32$, the identification accuracy of DLFD is $95.89 \%, 4.84 \%$ higher than that of LFD $91.05 \%$ within a normalized size $64 \times 640$. For the local partition area $16 \times 16$, the top identification accuracy of DLFD is always higher than that of LFD. For the local division area $8 \times 8$, the identification accuracy of DLFD increases to $98.95 \%$ when normalized to size $64 \times 128$ with a feature dimension 128 , while the identification accuracy of LFD decreases to $98.84 \%$. The enhancement is mainly because DLFD magnify the difference of intrinsic texture by taking the average fractal dimension as the reference, more precisely presenting the roughness of self-similarity texture.

However, for the local division area $4 \times 4$, DLFD do not reveal better performance than LFD. They are both $98.84 \%$ for the normalized $64 \times 64$ and $64 \times 128$. With the increase of normalized size, the recognition accuracy of DLFD keep 
stable, inferior to that of LFD. Small partition area result in more precise description in local texture of LFD, while the differences of LFD and the average (DLFD) of too small partitions magnify specific details, including noise which causes the deterioration of the performance. Moreover, large feature dimensions induced by too many small partitions burden computation and storage, leading to a low identification efficiency.

Table 1. Comparison of identification accuracies (\%) and feature dimensions of LFD and DLFD under different normalized size, local partition area.

\begin{tabular}{|c|c|c|c|c|c|c|}
\hline \multirow[t]{2}{*}{ Normalized size } & \multirow[t]{2}{*}{ Algorithms } & \multicolumn{5}{|c|}{ Local division Area } \\
\hline & & $64 \times 64$ & $32 \times 32$ & $16 \times 16$ & $8 \times 8$ & $4 \times 4$ \\
\hline \multirow{3}{*}{$64 \times 64$} & LFD & 26.11 & 62.00 & 96.53 & 98.63 & 98.84 \\
\hline & DLFD & ---- & 54.84 & 96.74 & 98.84 & 98.84 \\
\hline & Feature dimenison & 1 & 4 & 16 & 64 & 256 \\
\hline \multirow{3}{*}{$64 \times 128$} & LFD & 30.53 & 82.11 & 98.00 & 98.84 & 98.84 \\
\hline & DLFD & 18.00 & 85.89 & 98.53 & 98.95 & 98.84 \\
\hline & Feature dimenison & 2 & 8 & 32 & 128 & 512 \\
\hline \multirow{3}{*}{$64 \times 192$} & LFD & 44.42 & 88.21 & 97.16 & 98.84 & 98.84 \\
\hline & DLFD & 27.26 & 92.21 & 98.42 & 98.95 & 98.63 \\
\hline & Feature dimenison & 3 & 12 & 48 & 192 & 768 \\
\hline \multirow{3}{*}{$64 \times 256$} & LFD & 53.47 & 87.89 & 97.26 & 98.74 & 98.84 \\
\hline & DLFD & 46.32 & 92.84 & 98.74 & 98.95 & 98.63 \\
\hline & Feature dimenison & 4 & 16 & 64 & 256 & 1024 \\
\hline \multirow{3}{*}{$64 \times 320$} & LFD & 63.68 & 89.58 & 97.58 & 98.53 & 98.74 \\
\hline & DLFD & 63.05 & 94.74 & 98.42 & 98.74 & 98.63 \\
\hline & Feature dimenison & 5 & 20 & 80 & 320 & 1280 \\
\hline \multirow{3}{*}{$64 \times 384$} & LFD & 67.58 & 90.63 & 97.47 & 98.53 & 98.74 \\
\hline & DLFD & 71.58 & 94.74 & 98.53 & 98.84 & 98.63 \\
\hline & Feature dimenison & 6 & 24 & 96 & 384 & 1536 \\
\hline \multirow{3}{*}{$64 \times 448$} & LFD & 70.63 & 90.63 & 97.58 & 98.63 & 98.63 \\
\hline & DLFD & 76.42 & 95.05 & 98.53 & 98.84 & 98.63 \\
\hline & Feature dimenison & 7 & 28 & 112 & 448 & 1792 \\
\hline \multirow{3}{*}{$64 \times 512$} & LFD & 72.63 & 90.21 & 97.68 & 98.53 & 98.63 \\
\hline & DLFD & 82.00 & 95.26 & 98.53 & 98.95 & 98.63 \\
\hline & Feature dimenison & 8 & 32 & 128 & 512 & 2048 \\
\hline \multirow{3}{*}{$64 \times 576$} & LFD & 74.84 & 81.20 & 97.68 & 98.53 & 98.53 \\
\hline & DLFD & 83.47 & 95.58 & 98.53 & 98.84 & 98.63 \\
\hline & Feature dimenison & 9 & 36 & 144 & 576 & 2304 \\
\hline \multirow{3}{*}{$64 \times 640$} & LFD & 76.32 & 91.05 & 97.79 & 98.53 & 98.53 \\
\hline & DLFD & 84.63 & 95.89 & 98.63 & 98.95 & 98.63 \\
\hline & Feature dimenison & 10 & 40 & 160 & 640 & 2560 \\
\hline
\end{tabular}

Table 1 demonstrates a better overall performance of DLFD as compared to LFD. The top identification accuracy $98.95 \%$ of DLFD corresponds to $64 \times 128$ normalized size, $8 \times 8$ local division area and 128 feature dimension, selected as the optimum partition strategy in the following experiments. It can be concluded the ratios of length to width do not affect the identification accuracy obviously as observed, suggesting the identification performance is not closely related to the shape contours as we supposed. Comparatively, local block divisions influence the identification performance more obviously; too large local partition areas fail to describe the inner texture variation precisely, while too small partition areas magnify the noise and details in the mean time.

\subsubsection{Comparison of Different Seed Identification Algorithms}

To testify the effectiveness of DLFD, we compare the identification performance of different algorithms, including Fast Fourier Transform FFT_based DLFD, and appearance_based algorithm [4]. The FFT_based DLFD is to convolve FFT with the original image, and then we normalize the imaginary parts to integers of 0 and 255 for a convenient calculation for fractal dimension using DBC. Having divided the FFT transformed image into equal partitions, the DLFD of can be obtained according to the calculation equations of Section 3. The normalized size, division area, and the feature dimension of FFT_based DLFD were set $64 \times 128,8 \times 8$ and 128 , respectively, correspondence to the DLFD in 
4.2.1. No normalization and localization are required for appearance_based algorithm for the global contour and appearance features needed. Table 2 shows the identification accuracies of different algorithms.

Although FFT is one of the classical tools for image transformation to frequency domain, the identification rate of FFT_based DLFD is $93.16 \%, 5.43 \%$ inferior to that of DLFD based on the original image. The deterioration is mainly because the data type of FFT imaginary parts is float without obvious difference, while the FD obtained by DBC relies highly on the difference of minimum and maximum of gray scales within the local division area. The appearance_based algorithm [4] yields an identification rate of 78.84\% with only 12 feature components, including 6 morphological, 4 color and 2 textural seed characteristics. The deterioration demonstrates that holistic features can not represent the complete information of the image.

Table 2. The identification accuracies (\%) using and the corresponding feature dimension of different algorithms.

\begin{tabular}{|c|c|c|}
\hline Algorithms & Identification Accuracy & Feature Dimension \\
\hline DLFD & 98.59 & 128 \\
\hline LFD & 98.84 & 128 \\
\hline FFT+DLFD & 93.16 & 128 \\
\hline Appearance_based [4] & 78.84 & 12 \\
\hline
\end{tabular}

\section{CONCLUSION}

In this paper, we present a seed image based identification algorithm for gramineous grass. In the algorithm, fractal dimensions of all evenly divided local blocks were computed for textual description of the seed capsule. Thus average fractal dimension of all the blocks can be the basis for DLFD feature extraction. By subtracting the individual fractal dimension and the average, the feature vectors were composed of DLFD of all blocks with an emphasis on the contrast of the self-similarity of the seeds.

The algorithm was tested on a gramineous seed base composed of 19 similar species and compared to other classical seed identification approaches. The high accuracies achieved in this task suggested the effectiveness DLFD in intrinsic textual description of seed identification of textual analysis. The novelty lies in the extraction of the difference of local fractal dimension as the textural feature, taking advantage of the local self-similarity in seed images of gramineous grass, which is quite a new application area for pattern recognition. With more and more attention focused on forage identification and grassland monitor for the improvement of living conditions of human beings' ecosystem, computer vision is an essential tool to extend the application to a wider range and a more sophisticated extent.

\section{CONFLICT OF INTEREST}

The authors confirm that this article content has no conflict of interest.

\section{ACKNOWLEDGEMENTS}

This work is dedicated to Researcher $\mathrm{Zhu} \mathrm{Xu}$ and Dr. Feng Hao for their great contributions. This work was supported partly by the National Natural Science Foundation of China under Grant No.61582067 and No.31302017, the Postdoctoral Science Foundation of China No. 20100480370 and No. 201104179 , the National Natural Science Foundation of China under Grant No.61562067, the National Scientific and Technological Research Project 2013BAK05B01, the Ministry of water Resources Industry Special Project 201201008-022, the Basic Scientific Research Foundation for Central Public Research Institutes No. 1610332015007, and the Foundations of Inner Mongolia Agricultural University NDPYTD 210-9, BJ09-43.

\section{REFERENCES}

[1] A.J. Ishak, A. Hussain, and M.M. Mustafa, "Weed image classification using Gabor wavelet and gradient field distribution", Comput. Electron. Agric., vol. 66, pp. 53-61, 2009. [http://dx.doi.org/10.1016/j.compag.2008.12.003]

[2] F.K. Evert, and G. Polder, "GWAM. Van Der, C. Kempenaar, L. Lotz, "Real-time vision-based detection of Rumex obtusifolius in grassland", Eur. Weed Res. Soc. Weed Res., vol. 49, pp. 164-174, 2009. [http://dx.doi.org/10.1111/j.1365-3180.2008.00682.x]

[3] X.P. Burgos-Artizzu, A. Ribeiro, A. Tellaeche, G. Pajares, and C. Fernández-Quintanilla, "Analysis of natural images processing for the extraction of agricultural elements", Image Vis. Comput., vol. 28, pp. 138-149, 2010.

[http://dx.doi.org/10.1016/j.imavis.2009.05.009] 
[4] P.M. Granitto, H.D. Navone, P.F. Verdes, and H.A. Ceccatto, "Weed seeds identification by machine vision", Comput. Electron. Agric., vol. 33, pp. 91-103, 2002. [http://dx.doi.org/10.1016/S0168-1699(02)00004-2]

[5] P.M. Granitto, P.F. Verdes, and H.A. Ceccatto, "Large-scale investigation of weed seed identification by machine vision", Comput. Electron. Agric., vol. 47, pp. 15-24, 2005.

[http://dx.doi.org/10.1016/j.compag.2004.10.003]

[6] C. Shi, and G. Ji, "Study of recognition method of leguminous weed seeds image", In: Proceedings of International Workshop on Intelligent Systems Applications, 2009, pp. 1-4.

[http://dx.doi.org/10.1109/IWISA.2009.5073126]

[7] A. Pourreza, H. Pourreza, M. Abbaspour-Fard, and H. Sadrnia, "Identification of nine Iranian wheat seed varieties by textual analysis with image processing", Comput. Electron. Agric., vol. 83, pp. 102-108, 2012. [http://dx.doi.org/10.1016/j.compag.2012.02.005]

[8] J.B. Florindo, and O.M. Bruno, "Texture analysis by multi-resolution fractal descriptors", Expert Syst. Appl., vol. 40, pp. $4022-4028,2013$. [http://dx.doi.org/10.1016/j.eswa.2013.01.007]

[9] N. Sarkar, and B.B. Chaudhuri, "An efficient differential box-counting approach to compute fractal dimensions of image", IEEE Trans. Syst. Man Cybern., vol. 24, no. 1, pp. 115-120, 1994. [http://dx.doi.org/10.1109/21.259692]

[10] B. Mandelbrot, The Fractal geometry of nature, Freeman and Company: New York, 1982.

[11] P. Shanmugavadivu, and V. Sivakumar, "Fractal dimensions based texture analysis of digital images", Procedia Eng., vol. 38, pp. 2981-2986, 2012.

[http://dx.doi.org/10.1016/j.proeng.2012.06.348]

[12] W.N. Goncalces, and O.M. Bruno, "Combining fractal and deterministic walkers for texture analysis and classification", Pattern Recog., vol. 46, pp. 2953-2968, 2013. [http://dx.doi.org/10.1016/j.patcog.2013.03.012]

[13] J. Zou, Q. Ji, and G. Nagy, "A comparative study of local matching approach for face recognition", IEEE Trans. Image Process., vol. 16, no. 10 , pp. 2617-2628, 2007.

[http://dx.doi.org/10.1109/TIP.2007.904421] [PMID: 17926941]

[14] X. Pan, Y. Cen, Y. Ma, W. Yan, X. Gao, X. Liu, and G. Liu, "Identification of gramineous grass seeds using Gabor and locality preserving projections", Multimedia Tools Appl., 2016. [Online]. [http://dx.doi.org/10.1007/s11042-016-3424-0]

[15] R.C. Gonzalez, and R.E. Woods, Digital Image Processing., Prentice Hall, 2008.

(C) Pan et al.; Licensee Bentham Open.

This is an open access article licensed under the terms of the Creative Commons Attribution-Non-Commercial 4.0 International Public License (CC BY-NC 4.0) (https://creativecommons.org/licenses/by-nc/4.0/legalcode), which permits unrestricted, non-commercial use, distribution and reproduction in any medium, provided the work is properly cited. 